\section{POS0619 MODELLING OF DISEASE ACTIVITY IN PATIENTS WITH INFLAMMATORY ARTHROPATHIES TREATED WITH ETANERCEPT ORIGINATOR OR BIOSIMILAR AS FIRST-LINE BIOLOGIC IN AN AUSTRALIAN REAL- WORLD DATASET}

C. Deakin ${ }^{1}$, G. Littlejohn ${ }^{1,2}$, H. Griffiths ${ }^{1,3}$, T. Smith ${ }^{1}$, C. Osullivan ${ }^{1}$, P. Bird ${ }^{1,4}$ ${ }^{1}$ OPAL Rheumatology, Ltd., Sydney, Australia; ${ }^{2}$ Department of Medicine, Monash University, Clayton, Australia; ${ }^{3}$ Barwon Rheumatology, Services, Geelong, Australia; ${ }^{4}$ Department of Medicine, UNSW Sydney, Sydney, Australia

Background: The availability of biosimilars as non-proprietary versions of established biologic disease-modifying anti-rheumatic drugs (bDMARDs) is enabling greater access for patients with rheumatic diseases to effective medications at a lower cost. Since April 2017 both the originator and a biosimilar for etanercept (trade names Enbrel and Brenzys, respectively) have been available for use in Australia.

Objectives:

[1] To model effectiveness of etanercept originator or biosimilar in reducing Disease Activity Score 28-joint count C reactive protein (DAS28CRP) in patients with rheumatoid arthritis (RA), psoriatic arthritis (PsA) or ankylosing spondylitis (AS) treated with either drug as first-line bDMARD

[2] To describe persistence on etanercept originator or biosimilar as first-line bDMARD in patients with RA, PsA or AS

Methods: Clinical data were obtained from the Optimising Patient outcomes in Australian rheumatoLogy (OPAL) dataset, derived from electronic medical records. Eligible patients with RA, PsA or AS who initiated etanercept originator $(n=856)$ or biosimilar $(n=477)$ as first-line bDMARD between 1 April 2017 and 31 December 2020 were identified. Propensity score matching was performed to select patients on originator $(n=230)$ or biosimilar $(n=136)$ with similar characteristics in terms of diagnosis, disease duration, joint count, age, sex and concomitant medications. Data on clinical outcomes were recorded at 3 months after baseline, and then at 6-monthly intervals. Outcomes data that were missing at a recorded visit were imputed.

Effectiveness of the originator, relative to the biosimilar, for reducing DAS28CRP over time was modelled in the matched population using linear mixed models with both random intercepts and slopes to allow for individual heterogeneity, and weighting of individuals by inverse probability of treatment weights to ensure comparability between treatment groups. Time was modelled as a combination of linear, quadratic and cubic continuous variables.

Persistence on the originator or biosimilar was analysed using survival analysis (log-rank test).

Results: Reduction in DAS28CRP was associated with both time and etanercept originator treatment (Table 1). The conditional R-squared for the model was 0.31. The average predicted DAS28CRP at baseline, 3 months, 6 months, 9 months and 12 months were 4.0 and 4.4, 3.1 and 3.4, 2.6 and 2.8, 2.3 and 2.6, and 2.2 and 2.4 for the originator and biosimilar, respectively, indicating a clinically meaningful effect of time for patients on either drug and an additional modest improvement for patients on the originator.

Median time to $50 \%$ of patients stopping treatment was 25.5 months for the originator and 24.1 months for the biosimilar $(p=0.53)$. An adverse event was the reason for discontinuing treatment in 33 patients $(14.5 \%)$ on the originator and 18 patients $(12.9 \%)$ on the biosimilar.

Conclusion: Analysis using a large national real-world dataset showed treatment with either the etanercept originator or the biosimilar was associated with a reduction in DAS28CRP over time, with the originator being associated with a further modest reduction in DAS28CRP that was not clinically significant. Persistence on treatment was not different between the two drugs.

Table 1. Model summary for prediction of DAS28CRP using time and etanercept originator (relative to biosimilar)

\begin{tabular}{lccc}
\hline Fixed Effect & Estimate & $95 \%$ Confidence Interval & $p$-value \\
\hline Time (linear) & 0.90 & $0.89,0.91$ & $1.5 \mathrm{e}-63$ \\
Time (quadratic) & 1.01 & $1.00,1.01$ & $1.3 \mathrm{e}-33$ \\
Time (cubic) & 1.00 & $1.00,1.00$ & $7.1 \mathrm{e}-23$ \\
Originator & 0.91 & $0.86,0.96$ & 0.0013 \\
\hline
\end{tabular}

Acknowledgements: The authors acknowledge the members of OPAL Rheumatology Ltd and their patients for providing clinical data for this study, and Software4Specialists Pty Ltd for providing the Audit4 platform.

Supported in part by a research grant from Investigator-Initiated Studies Program of Merck \& Co Inc, Kenilworth, NJ, USA. The opinions expressed in this paper are those of the authors and do not necessarily represent those of Merck \& Co Inc, Kenilworth, NJ, USA

Disclosure of Interests: Claire Deakin: None declared, Geoff Littlejohn Consultant of: Over the last 5 years Geoffrey Littlejohn has received educational grants and consulting fees from AbbVie, Bristol Myers Squibb, Eli Lilly, Gilead, Novartis, Pfizer, Janssen, Sandoz, Sanofi and Seqirus., Hedley Griffiths Consultant of: AbbVie, Gilead, Novartis and Lilly., Tegan Smith: None declared, Catherine OSullivan: None declared, Paul Bird Speakers bureau: Eli Lilly, abbvie, pfizer, BMS, UCB, Gilead, Novartis

DOI: 10.1136/annrheumdis-2021-eular.2265

\section{POS0620 \\ TREATMENT PATHWAYS OF RHEUMATOID ARTHRITIS PATIENTS LEADING TO BIOLOGIC THERAPY VISUALIZED IN A SANKEY DIAGRAM}

T. Coppes $^{1}$, N. Jessurun ${ }^{1}$, J. Jansen ${ }^{1}$, K. Velthuis ${ }^{1}$, P. ten Kloster ${ }^{2,3}$, H. Vonkeman ${ }^{3,4}$. ${ }^{1}$ Netherlands Pharmacovigilance Centre Lareb, Monitoring 's-Hertogenbosch, Netherlands; ${ }^{2}$ Transparency in Healthcare B. V., Hengelo, Netherlands; ${ }^{3}$ University of Twente, Psychology, Health \& Technology, Enschede, Netherlands; ${ }^{4}$ Medisch Spectrum Twente, Rheumatology and Clinical Immunology, Enschede, Netherlands

Background: Treatment of rheumatoid arthritis (RA) has changed in the past 20 years due to the arrival of biologic disease-modifying antirheumatic drugs (bDMARDs) and the application of treat to target strategies. Many different combinations of conventional synthetic (cs)DMARDS and bDMARDS are being applied in daily practice. It is difficult to visualize and understand all the different treatment pathways that are sequentially being used over longer periods of time in a real-world setting. We therefore investigated whether a Sankey diagram is a suitable tool to study and visualize which treatment pathways exist and to what extent a RA treatment protocol is being followed.

Objectives: To illustrate the treatment pathways over longer periods of time in a cohort of early RA patients and to assess adherence to the treatment protocol using Sankey diagrams.

Methods: This single-centre retrospective, observational cohort study used data from the DREAM-RA registry. Patients were treated according to a treat to target strategy with a step-up DMARD treatment protocol when remission was not achieved, initial steps were starting with MTX $15 \mathrm{mg} / \mathrm{wk}$, week 8 MTX $25 \mathrm{mg} /$

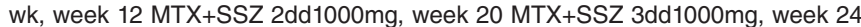
MTX+adalimumab 40mg/2wk, week $36 \mathrm{MTX}+$ adalimumab $40 \mathrm{mg} / \mathrm{wk}$. Although the protocol met full consensus, adherence to the protocol in individual patients was at the discretion of the treating rheumatologist. In this study, patients were included if they received a continuous treatment with a conventional synthetic or biologic DMARD between 1 January 2002 and 30 April 2020. During treatment, corticosteroids per protocol were allowed but not considered as an individual treatment. Evaluated outcomes included: the consecutive treatments that patients followed including start- and stop-date of treatments, the proportion of patients that received bDMARDs, the number of switches until first bDMARD, and time to first bDMARD. Furthermore, the lower limit of adherence to the protocol was estimated by considering all patients ( $\%$ of total) treated according to the protocol. This information was determined by verifying whether the patient was being treated according to the protocol after each switch.

Results: A total of 372 patients were included in this study (Table 1). The mean overall follow-up time of the cohort was $8.83( \pm 3.59)$ years. The follow-up time for the first 4 treatments, depicted in the Sankey diagram was $6.28( \pm 3.31)$ years. At least $45(12 \%)$ patients started with a bDMARD before all previous protoco steps were followed. At the start of treatment, $81 \%$ of the patients were treated according to the protocol, this was reduced to $28 \%$ after one switch. The lower limit of adherence to the predefined protocol after 3 switches were roughly $5 \%$ of all patients.

Table 1. Patient characteristics of all included patients

\begin{tabular}{lc}
\hline & All patients $(\mathrm{n}=372)$ \\
\hline Gender, female, $\mathrm{n}(\%)$ & $246(66.1)$ \\
Age, year, mean \pm SD & $67.9 \pm 13.61$ \\
Overall follow-up, years \pm SD & $8.83 \pm 3.59$ \\
Follow-up first 4 treatments, years \pm SD & $6.28 \pm 3.31$ \\
Baseline DAS-28 score, mean \pm SD & $3.60 \pm 1.41$ \\
Rheumatoid factor positive, $\mathrm{n}(\%)$ & $336(90.3)$ \\
Patients who eventually received a bDMARD, $\mathrm{n}(\%)$ & $108(29.0)$ \\
Number of switches until first bDMARD, mean \pm SD & $2.7 \pm 1.41$ \\
Time to first bDMARD, years \pm SD & $3.66 \pm 3.00$ \\
\hline
\end{tabular}




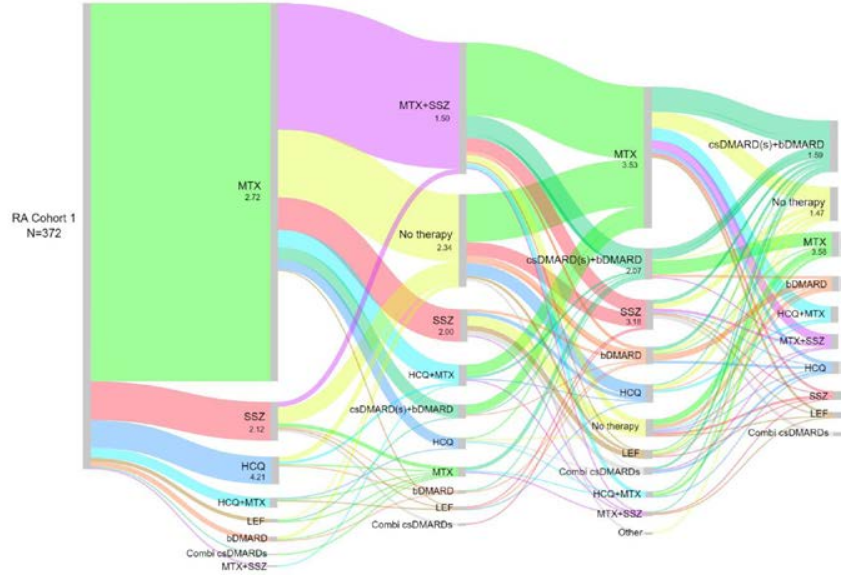

Figure 1. Sankey diagram of the treatment pathway of the first 3 switches of RA patients. The average duration of treatment of a flow is displayed in years if the flow included more than 20 patients. (MTX= methotrexate; $S S Z=$ sulfasalazine; $\mathrm{HCQ}=$ hydroxychloroquine; $\mathrm{LEF}=$ leflunomide; $b D M A R D=$ monotherapy bDMARD; Combi csDMARDs= combination therapy of csDMARDS; $\operatorname{csDMARD}(\mathrm{s})+\mathrm{bDMARD}=$ combination therapy of one or two csDMARD(s) + a bDMARD; No therapy $=$ no treatment received $>3$ months; Other $=$ medication that is not a (cs)(b)DMARD)

Conclusion: Sankey diagramming can be used to illustrate complex real-world treatment data of a treat to target cohort of RA patients. Treatment protocol adherence can be assessed with the help of a Sankey diagram. After 3 switches, the lower limit of adherence to the protocol was roughly $5 \%$.

Disclosure of Interests: Tristan Coppes: None declared, Naomi Jessurun: None declared, Jurriaan Jansen: None declared, Kimberly Velthuis: None declared, Peter ten Klooster: None declared, Harald Vonkeman Consultant of: BMS, Celgene, Celltrion, Galapagos, Gilead, Janssen-Cilag, Lilly, Novartis, Pfizer, Sanofi-Genzyme, Grant/research support from: Abbvie DOI: 10.1136/annrheumdis-2021-eular.2299

\section{POS0621 DISEASE-SPECIFIC ADVERSE DRUG REACTION PROFILES OF ADALIMUMAB AND ETANERCEPT AS REPORTED BY IMMUNE-MEDIATED INFLAMMATORY DISEASE PATIENTS}

L. Roest ${ }^{1}$, L. Kosse ${ }^{1}$, J. van Lint ${ }^{1}$, J. Scholl ${ }^{1}$, M. van Doorn ${ }^{2}$, S. Tas ${ }^{3}$, M. Nurmohamed ${ }^{4}$, H. Vonkeman ${ }^{5,6}$, R. Hebing ${ }^{7}$, P. Spuls ${ }^{8}$, F. Hoentjen ${ }^{9}$, E. van Puijenbroek ${ }^{10,11}$, B. van den Bemt ${ }^{10,12}$, N. Jessurun ${ }^{1} .{ }^{1}$ Netherlands Pharmacovigilance Centre Lareb, Department of Monitoring, 's-Hertogenbosch, Netherlands; ${ }^{2}$ Erasmus MC, Department of Dermatology, Rotterdam, Netherlands; ${ }^{3}$ Amsterdam UMC, Location Academic Medical Center, University of Amsterdam, Amsterdam Infection \& Immunity Institute and Amsterdam Rheumatology \& Immunology Center (ARC), Department of Rheumatology \& Clinical Immunology, Amsterdam, Netherlands; ${ }^{4}$ Reade and Amsterdam Rheumatology \& Immunology Center (ARC), Department of Rheumatology, Amsterdam, Netherlands; ${ }^{5} \mathrm{Health} \&$ Technology, University of Twente, Department of Psychology, Enschede, Netherlands; ${ }^{6}$ Medisch Spectrum Twente, Department of Rheumatology, Enschede, Netherlands; ${ }^{7}$ Amsterdam Rheumatology and Immunology Center Location Reade, Department of Pharmacy, Amsterdam, Netherlands; ${ }^{8}$ Amsterdam UMC, Amsterdam Public Health, Immunity and Infections, University of Amsterdam, Department of Dermatology, Amsterdam, Netherlands; ${ }^{9}$ Radboud UMC, Department of Gastroenterology, Nijmegen, Netherlands; ${ }^{10}$ Sint Maartenskliniek, Department of Pharmacy, Nijmegen, Netherlands; ${ }^{11}$ Netherlands Pharmacovigilance Centre Lareb, Department of Signal Detection, 's-Hertogenbosch, Netherlands; ${ }^{12}$ Radboud UMC, Department of Pharmacy, Radboud UMC, Netherlands

Background: Information on adverse drug reactions (ADRs) is generally clustered for all indications of a drug in the patient information leaflet. However, previous research has shown that participants of the Dutch Biologic Monitor (DBM) that use a biologic for their immune-mediated inflammatory disease (IMID) prefer to receive ADR information tailored to their own biologic an IMID (1). Currently, it is unclear whether the ADR profile of a specific biologic may differ between patients with different IMIDs, which would be vital information for health care providers (HCPs) in their patient guidance.

Objectives: To determine whether the profiles of ADRs attributed to adalimumab (ADA) and etanercept (ETN) reported by patients in the DBM differ between IMIDs.

Methods: The DBM is a prospective cohort event monitoring system for patient-reported ADRs attributed to biologics (2). Study data was extracted from the DBM for the period Jan 2017 - Oct 2020. ADRs were coded according to their corresponding Preferred Term (PT) following MedDRA terminology. Unique PTs were selected per participant and grouped under System Organ Classes (SOCs) (Figure 1) for ADA and ETN. SOCs contributing for $<1 \%$ to the total number of reported ADRs were grouped as 'other'. Participants with more than one of the included IMIDs, i.e. Psoriatic Arthritis (PsA), Inflammatory Bowel Disease (IBD, i.e. Crohn's disease and ulcerative colitis), rheumatoid arthritis (RA), and axial spondyloarthritis (axSpA) including Ankylosing Spondylitis (AS), were excluded. Differences in ADR profiles between IMIDs were tested using the Fisher-Freeman-Halton's Exact Test with Monte Carlo simulation. SOCs of interest were separately tested with the Fisher-Freeman-Halton's Exact Test (no simulation) and subsequently corrected for multiple comparisons using the Benjamini-Hochberg (BH) correction.

Results: A total of 572 ADR reports from 218 participants using ADA and 450 ADR reports from 185 participants using ETN were analyzed (Table 1).

Overall, a statistically significant difference in patient-reported ADR profile between the assessed indications was found for ADA ( $p=0.011)$, but not for ETN $(p=0.057)$. The following separate tests for selected SOCs of interest showed a significant difference in the frequencies of 'respiratory, thoracic and mediastinal disorders' and 'musculoskeletal and connective tissue disorders' between the different IMIDs for ADA after BH correction, but none for ETN.
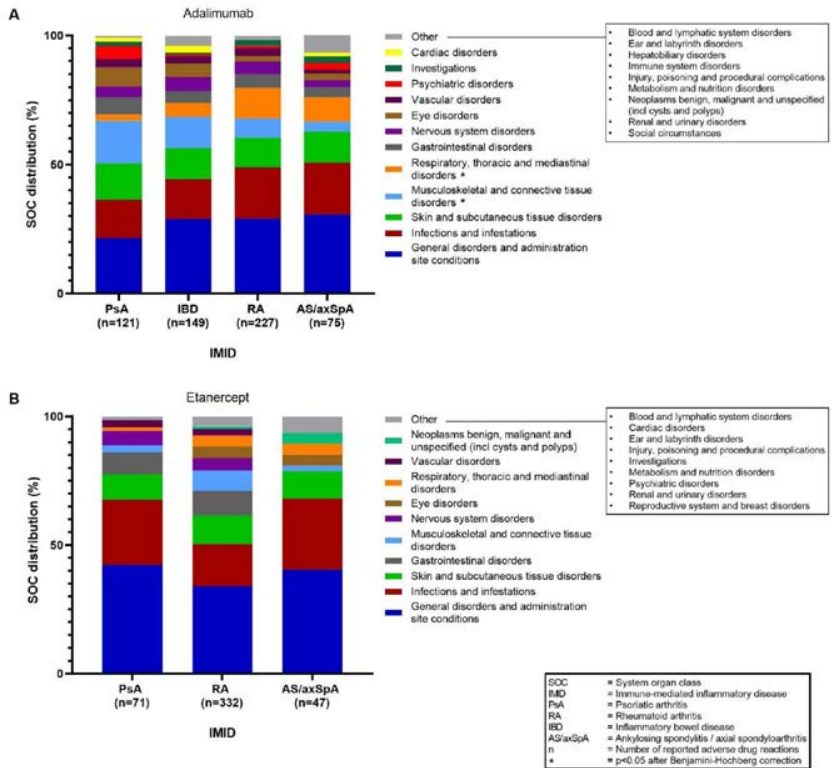

Figure 1. The disease-specific patient-reported ADR profile of ADA (A) and ETN (B) in IMID patients resulting from the Dutch Biologic Monitor

Table 1. Respondent characteristics.

\begin{tabular}{lcccc}
\hline \multirow{2}{*}{ Characteristics } & \multicolumn{3}{c}{ ADA } & \multicolumn{2}{c}{ ETN } \\
\cline { 2 - 5 } & $n=218$ & $\%$ & $n=185$ & $\%$ \\
\hline Female gender, $n$ (\%) & 140 & 64.2 & 129 & 69.7 \\
Median age (IQR), years & 56.0 & $(46.0-64.0)$ & 58.0 & $(48.0-66.0)$ \\
ADR reports & 572 & 100.0 & 450 & 100.0 \\
Indication for biologic therapy & & & & \\
$\quad$ Rheumatoid arthritis & 90 & 41.3 & 127 & 68.6 \\
Psoriatic arthritis & 46 & 21.1 & 35 & 18.9 \\
Ankylosing spondylitis/axSpA & 32 & 14.7 & 23 & 12.4 \\
IBD & 50 & 22.9 & 0 & 0.0 \\
Combination therapy & & & & \\
$\quad$ Methotrexate & 63 & 30.3 & 70 & 40.2 \\
Corticosteroids & 25 & 12.0 & 21 & 12.1 \\
Thiopurines & 18 & 8.7 & 1 & 0.6 \\
$\quad$ No combination therapy & 87 & 41.8 & 59 & 33.9 \\
Other & 31 & 14.9 & 48 & 28.2 \\
\hline
\end{tabular}

IQR: interquartile range; IBD: inflammatory bowel disease; axSpA: axial spondyloarthritis. ${ }^{a} \mathrm{IBD}$ includes Crohn's disease and ulcerative colitis. ${ }^{\mathrm{b}}$ The overall percentage exceeds $100 \%$ since patients can have a combination therapy consisting of one or more drugs.

Conclusion: Although only ADA shows a statistically significant difference in ADR profile between different IMIDs, more research with a larger sample size might show similar results for ETN. Furthermore, explanations for the differences found, such as disease-drug interactions, must be examined. This would help HCPs in providing disease-specific information and patient guidance. 